\section{$\underset{\substack{\text { hommes } \\ \text { \& migrations }}}{ }$}

\section{Hommes \& migrations}

Revue française de référence sur les dynamiques

migratoires

1306 | 2014

Ecriture et migration

\title{
La Légion étrangère, une particularité française
}

\section{Valérie Esclangon-Morin}

\section{CpenEdition \\ Journals}

\section{Édition électronique}

URL : http://journals.openedition.org/hommesmigrations/2844

DOI : 10.4000/hommesmigrations.2844

ISSN : 2262-3353

\section{Éditeur}

Musée national de l'histoire de l'immigration

\section{Édition imprimée}

Date de publication : 1 avril 2014

Pagination : 133-137

ISBN : 978-2-919040-27-8

ISSN : $1142-852 X$

\section{Référence électronique}

Valérie Esclangon-Morin, « La Légion étrangère, une particularité française », Hommes \& migrations [En ligne], 1306 | 2014, mis en ligne le 06 août 2014, consulté le 22 septembre 2020. URL : http://

journals.openedition.org/hommesmigrations/2844; DOl : https://doi.org/10.4000/

hommesmigrations.2844 


\section{CHRONIQUES DE GUERRE}

\section{LA LÉGION ÉTRANGÈRE, UNE PARTICULARITÉ FRANÇAISE}

VALÉRIE ESCLANGON-MORIN, historienne, professeur-relai au Musée de l'histoire de l'immigration

"Qui sait si l'inconnu qui dort sous l'arche immense, Mêlant sa gloire épique aux orgueils du passé N'est pas cet étranger devenu fils de France Non par le sang reçu mais par le sang versé ?" Pascal Bonetti, 1920.

\section{"Étrangère! non pas! ô France, depuis quand} Le baptême du sang n'est-il plus un baptême? Quidonc vous renierait Français sans un blasphème, Martyrs de Camaron, héros de Tuyen-Quan?" Henri Richardot (Dachères), 1912.

$D^{\prime}$ rôle de troupe ces légionnaires, héritage des bataillons étrangers levés par les rois de France puis par Napoléon. La Restauration continue ce recrutement particulier et la conquête de l'Algérie achève d'en faire une troupe à part entière. Ce sera la Légion étrangère, constituée en 1831 pour répondre aux nouvelles exigences de la conquête coloniale.

Quels sont les objectifs de cette Légion étrangère ? Quels sont les étrangers qui la composent et quelles sont leurs motivations pour entrer dans ce corps spécifique ? L'analyse de ces motifs nous incite à nous pencher sur les raisons de la création de la Légion, sur la constitution de cette troupe mais aussi sur la création du mythe de la Légion comme modèle d'intégration et d'adhésion aux valeurs universelles de la France.

\section{Une réponse aux besoins des conquêtes coloniales}

Au XIXe siècle, le développement des guerres sur les terrains extérieurs à la France (colonies africaines et asiatiques, Mexique, Crimée) rend indispensable la levée de nouvelles troupes. En complément des troupes coloniales et des troupes indigènes de l'armée d'Afrique, la constitution de corps de volontaires étrangers devient une évidence.

Cette troupe est envoyée dans tous les théâtres d'opérations où elle s'illustre souvent. Ainsi, à Camerone en avril 1863, 60 légionnaires résistèrent héroïquement à 2000 Mexicains. La Légion y gagne ses heures de gloire et son mythe fondateur. Au fur et à mesure des expéditions, la Légion devient une troupe de choc qui a la réputation de combattre jusqu'à la mort.

Elle est aussi utilisée à d'autres fins. Elle se fait bâtisseuse, les légionnaires construisant des routes et des villages de colonisation, notamment en Afrique. Il existe toujours au niveau de la Légion un corps de pionniers, reconnaissable à leur tenue, composée de la hache, du tablier de cuir et de la barbe.

L’Afrique reste le creuset de la Légion. La conquête algérienne a généré son existence et c'est en Algérie, à Sidi-Bel-Abbès (Sud oranais), qu'a longtemps été le cœur de la Légion. Jusqu'en 1962, elle 


\section{CHRONIQUES DE GUERRE}

y a ses quartiers, son centre de recrutement et son centre de formation, sa prison aussi. Après l'indépendance algérienne, en 1962, la Légion se replie à Aubagne dans le sud de la France.

La France s'enorgueillit de cette troupe de choc qu'elle malmène pourtant, l'envoyant dans les expéditions les plus meurtrières. La Légion a payé un très lourd tribut lors des deux guerres coloniales d'Indochine et d'Algérie. Aujourd'hui encore, elle est de tous les combats en Afrique et au Moyen-Orient.

\section{Devenir légionnaire}

Pourquoi servir dans la Légion? Quelle est l'attractivité de ce corps si particulier? Est-ce réellement, comme le dit Pascal Bonetti, pour devenir français "par le sang versé" ? La naturalisation française, à laquelle le légionnaire a droit à partir de cing ans de service, est-elle réellement un graal que l'on vient chercher du monde entier?

L'analyse de l'histoire des légionnaires semble brouiller un peu cette belle image. Le nombre important de désertions aussi, ces hommes n'hésitant pas à partir lorsqu'ils estimaient ne plus trouver ce qu'ils étaient venus chercher.

Certes, il y a bien eu l'enthousiasme des milliers de volontaires étrangers se rendant en masse dans les bureaux de recrutement en août 1914, demandant à défendre la France et ses valeurs contre l'agression allemande. Ces étrangers ont été recrutés à partir d'août 1914 sous l'appellation d'“engagés volontaires pour la durée de la guerre". C'étaient essentiellement des intellectuels francophiles et/ou vivant en France depuis longtemps. L'appel lancé par Blaise Cendrars et d'autres intellectuels résume bien leur motivation : "L'heure est grave. Tout homme digne de ce nom doit aujourd'hui agir, doit se défendre de rester inactif au milieu de la plus formidable conflagration que l'Histoire n'ait jamais connue. Toute hésitation serait un crime. Point de paroles, donc des actes. (...) Des étrangers, amis de la France, qui pendant leur séjour sur le territoire ont appris à l'aimer et à la chérir comme une seconde patrie, sentent le besoin impérieux de lui offrir leur bras'." Suite à cet appel, plus de 8 ooo candidats à l'engagement pour la durée de la guerre se présentent le 3 août dans les permanences que l'association Les Amitiés françaises a ouvertes. Les demandes d'engagement sont adressées au ministère de la Guerre. D'autres appels suivent : celui d'Américains mené par Georges Casmèze, celui des juifs ou des Arméniens, des Syriens ou encore des Grecs². Pour certaines communautés, cet engagement est l'occasion de demander la création de corps d'armée composés de membres de la même nation, comme c'est le cas pour les 300 Polonais - ils composent, en 1914, une compagnie du $1^{\text {er }}$ régiment étranger, qui possède même ses drapeaux officieux avec l'aigle polonais ${ }^{3}$ ou encore pour les Italiens qui servent dans la Légion garibaldienne, composant le $4^{\mathrm{e}}$ régiment étranger de la Légion étrangère ${ }^{4}$. Au total, plus de 32000 hommes - représentant plus de 50 nationalités - s'engagent comme volontaires étrangers pour la durée de la guerres.

\footnotetext{
1. Appel du $1^{\text {er }}$ août signé Cendrars, Sarlius, Csaki, Kaplan, Berr, Oknosky, Dibroski, Isbicki, Schoumoff, Roldireff, Kozline, Esen, Lioschits, Frisendahl, Israilivitch, Vertepoff, Canudo. 2. Appels reproduits dans l'ouvrage de Jean-Paul Mahuault, Engagés volontaires à la Légion étrangère pour la durée de la guerre, Monts, éd. Grancher, 2013, pp. 82-92. 3. Il existe un monument aux morts commémorant ces volontaires polonais morts pour la France à Neuville-Saint-Vaast, bâti en 1929, "à la mémoire des volontaires polonais qui, le 9 mai 1915 , se sont portés à l'assaut de la cote 140 et sont tombés pour la résurrection de la Pologne et la victoire de la France \#. 4. Ils furent 2354 volontaires dans cette légion garibaldienne, dont Lazare Ponticelli, dernier poilu français décédé en 2008 (les légionnaires ont d’ailleurs porté son cercueil). Ce régiment est dissous le 5 mars 1915 et la plupart des “garibaldiens" ont ensuite intégré les rangs de l'armée italienne, l'Italie entrant en guerre à son tour le 24 mai 1915. 5. Le ministère de la Guerre décide de n'ouvrir les recrutements qu'à partir du 21 août (soit le $20^{e}$ jour de mobilisation) pour éviter l'enthousiasme des premiers jours. Les bureaux de recrutement des Invalides sont pourtant débordés à cette date. Plusieurs bureaux doivent être créés à Paris. Du 21 août 1914 au 1 ${ }^{\mathrm{er}}$ avril 1915, 25834 engagements sont signés, dont 6500 Alsaciens-Lorrains, 3393 Russes, 600 Arméniens. Chiffres extraits de l'ouvrage de Jean-Paul Mahuault, op. cit, p. 93.
} 
Les raisons de leur engagement sont bien différentes de celles des légionnaires. Leur réversion dans les régiments de la Légion étrangère à partir de 1915 est d'ailleurs l'occasion pour Blaise Cendrars - lui-même engagé volontaire pour la durée de la guerre - de narrer ce choc des cultures: "L'arrivée de ces têtes brûlées de retour d'Afrique, de ces survivants de je ne sais quelles infernales campagnes coloniales, de je ne sais quelles criminelles expéditions punitives dans l'extrême sud, qui étaient montés en ligne comme nous commencions à crever de misère et de honte dans les tranchées, nous fit un bien à tous, car chacun de ces damnés, ça se voyait, était moralement dépouillé de tout, orgueilleux et solitaire; et c'est cet abominable moral d'hommes d'action, pour ne pas dire d'hommes de main, ou encore de héros désenchantés et revenus de tout, beaucoup plus que leur esprit de corps, auquel ils semblaient si manifestement, si exagérément tenir, qui nous servit d'exemple, à nous, jeunes écervelés, enthousiastes ou je m'en-fichistes (...) dont se composait notre III régiment de marche de la Légion étrangère, le régiment le plus parisien de tous les régiments de l'armée française, et le plus intellectuel de tous (...)."."

En dehors de ces périodes de guerre, qui sont ces volontaires étrangers désirant entrer dans la Légion et quelles sont leurs motivations ? Devenir français n'est certes pas leur priorité première. La Légion propose autre chose : elle est une famille d'accueil pour des êtres brisés par la vie ou en quête d'une nouvelle patrie. Mais cette patrie n'est pas la France, c'est la Légion : "Legio patria nostra" est bien la devise des légionnaires.

Elle absorbe les hommes en quête d'un nouvel idéal ou fuyant une vie éprouvante. Elle propose l'ultime possibilité, celle de l'effacement. Les légionnaires deviennent des "hommes sans nom", des hommes sans passé, sans patrie. "Ce corps a pour vocation d'accueillir des hommes sans racines qui veulent tirer un trait sur leur passé", dit Hélie de Saint-Marc, officier français de la Légion. Ils peuvent se racheter - ou pas - de leur vie d'avant. Ils entrent dans la famille: "Chaque légionnaire est ton frère d'armes, quelles que soient sa nationalité, sa race ou sa religion. Tu lui manifestes toujours la solidarité étroite qui doit unir les membres d'une même famille", indique le $2^{\mathrm{e}}$ article du code d'honneur du légionnaire.

\section{Une légion franco-germanique?}

La Légion est d'abord le creuset des hommes perdus, des apatrides, des vaincus de l'histoire. Pour cela, on y parle beaucoup la langue des exilés: le yiddish et le polonais au XIXe siècle, le russe dans les années 1920, l'italien et l'espagnol dans les années 1930... Mais la langue la plus parlée dans la Légion reste l'allemand. Entre 1830 et 1961, sur 600 ooo légionnaires ayant servi dans la Légion, 210000 sont allemands, 60 ooo italiens, 50 ooo belges et Français, 40 ooo espagnols et 30 ooo suisses. Ils représentent même 60 \% des régiments étrangers entre 1945 et 1955, largement employés lors des guerres coloniales (Madagascar, Indochine et Algérie).

Ce choix de la Légion par de nombreux jeunes Allemands reste à la fois un mystère et une source de
La Légion absorbe les hommes en quête d'un nouvel idéal ou fuyant une vie éprouvante. Elle propose l'ultime possibilité, celle de l'effacement. Les légionnaires deviennent des "hommes sans nom", des hommes sans passé, sans patrie. et l'Allemagne. Désir d'aventure ? Besoin de se mettre à l'abri du besoin ? Ou'est-ce qui pousse ces jeunes Allemands à s'engager pour une patrie 


\section{CHRONIQUES DE GUERRE}

qui est, par trois fois, son ennemi ? À l'inverse, pourquoi l'armée française accepte-t-elle de recevoir un nombre aussi conséquent de ces potentiels ennemis?

Une première réponse est que, dans la Légion, on oublie sa nationalité, on est avant tout légionnaire. D'autre part, l'état-major de la Légion aime ces nationaux qui ont le sens de la discipline et incite donc au recrutement de légionnaires allemands (à l'exception de la période 1870 et 1880 , où le sentiment anti-allemand est encore vif). Enfin, de 1871 à 1914, une grande partie des Allemands recrutés étaient des Alsaciens-Lorrains désirant fuir la conscription allemande ${ }^{7}$. C'est bien le seul réel exemple de motivation patriotique ${ }^{8}$. La loi du 5 août 1914 stipule d'ailleurs que tout Alsacien-Lorrain contractant un engagement volontaire au titre d'un des régiments étrangers recouvre la nationa-

La grande majorité des légionnaires ne demandent pas la nationalité française.

Si la France s'enorgueillit

de ce corps spécifique,

c'est en vertu de l'illusion

qu'elle s'est forgée

d'une nation qui attire

les étrangers du monde entier,

y compris pour combattre à ses côtés.

lité française. Ils sont 11854 à s'engager au $1^{\text {er }}$ avril 1915, soit plus du tiers des engagés volontaires dans l'armée française.

L'envie d'ailleurs peut aussi expliquer cette motivation. La loi du 9 mars 1831 stipulait que la Légion ne pouvait être employée qu'en dehors du territoire national. Pas question donc de s'impliquer dans des conflits nationaux. Cette loi n'étant cependant valable qu'en temps de paix, la Légion s'est trouvée engagée dans le premier conflit mondial. La France respecte pourtant la convention de La Haye qui stipule l'interdiction faite "à un belligérant de forcer des nationaux de la partie adverse à prendre part aux opérations de guerre dirigées contre leur pays". Des camps d'internement sont donc ouverts pour les légionnaires allemands, mais les meilleurs sont regroupés dans les bataillons des deux régiments de marche créés à la demande du général Lyautey pour assurer la sécurité du protectorat du Maroc, dégarni de ses autres troupes envoyées au front. De même, au lendemain de la guerre, la Légion du Maroc offre la possibilité, pour de nombreux Allemands, de s'engager au service de l'ancien ennemi ; la France - et Lyautey au Maroc - encourage d'ailleurs ce recrutement, notamment en ouvrant des bureaux de recrutement dans la partie occupée par la France en Rhénanie9.

Les différents gouvernements allemands ont pourtant tout essayé pour dissuader leurs jeunes de s'engager dans la Légion. À mesure que croissait le nationalisme, le départ de ces jeunes vers une armée étrangère choquait l'opinion publique allemande. Les nazis ont été les plus virulents. Sous le III Reich, toute allusion à la Légion est prohibée. On crée un camp de concentration à Kislau (pays de Bade) pour "accueillir" les légionnaires en fin de contrat. Au printemps 1939, la nationalité allemande est même retirée aux légionnaires. Pourtant, la Légion, quoique française, reste un idéal pour des jeunes soldats allemands déboussolés par la défaite en 1918 comme en 1945. En 1945, elle sert d'accueil pour de nombreux Allemands venant des anciennes régions orientales de l'Allemagne, devenues polonaises ou tchèques. Elle permet aussi aux anciens nazis ayant échappé aux foudres de la justice, de se refaire une identité. Le jeune officier Hélie de Saint-Marc, ancien résistant et réchappé des camps de concentration nazis, se retrouve ainsi dans un bateau pour 
I'Indochine au milieu d'hommes qu'il a combattus. Ce qu'il en retient, c'est avant tout la capacité qu'a la Légion à d'effacer : "Les hommes avaient mis entre parenthèses leur nom, leur famille, leurs racines, leur nationalité. Ils sétaient volontairement dépouillés de tout ce qui fait l'apparence sociale. Devant nous, ils étaient nus. La règle tacite était de ne jamais parler du passé. Nous la respections tous, conscients que la moindre curiosité pouvait être une offense. Je partageais avec eux la vision de trop de morts. Comme moi, ils essayaient de vivre avec le souvenir des engloutis"."

\section{La Légion, creuset de l'intégration à la française ?}

Sont-ils pour autant "français par le sang versé" ? Pas si sûr. Leurs noms n'apparaissent pas sur les monuments aux morts des villages de France et il faut attendre la loi du 29 décembre 1999 pour que la France accorde de droit la nationalité française aux légionnaires blessés au combat. Ils n'obtiennent pour toute intégration qu'une carte de résident au bout de trois ans de service à condition d'avoir auparavant obtenu un certificat de bonne conduite. Si le légionnaire veut devenir français, la procédure de naturalisation lui est facilitée : il est dispensé de prouver les cinq années de résidence en France et de passer une épreuve de langue, et la décision est quasiment toujours favorable.

Néanmoins, l'intégration par le sang versé reste un mythe largement entretenu par la France. La grande majorité des légionnaires ne demandent pas la nationalité française. Si la France s'enorgueillit de ce corps spécifique, c'est en vertu de l'il- lusion qu'elle s'est forgée d'une nation qui attire les étrangers du monde entier, y compris pour combattre à ses côtés. C'est pourquoi la Légion est à l'honneur aujourd'hui lors des défilés du 14 Juillet. Le défilé des compagnies de sapeurspionniers, habillés du tablier de cuir et portant la hache, perpétue le mythe du soldat bâtisseur. Ce sont les héritiers des soldats de l'an II, s'enthousiasmant pour une France révolutionnaire aux accents des Lumières. Ce sont les héritiers des soldats de la Grande Armée, conquérant les pays européens avec le Code civil.

Sans doute l'union entre la France et sa Légion repose-t-elle sur une vaste supercherie. La Légion offre une famille et un idéal à des milliers d'hommes dans le monde, une raison d'espérer à une République française en quête de modèles d'intégration. Mais laisser croire que la Légion est l'exemple parfait de l'intégration à la française relève de la construction d'un mythe national. 